\title{
Integrated Approach for Self-Balancing Production Line with Multiple Parts
}

\author{
Daisuke Hirotani ${ }^{1}$, Katsumi Morikawa ${ }^{1}$ and Katsuhiko Takahashi ${ }^{1}$ \\ 1 Aritificial Complex Systems Engineering, Graduate School of \\ Engineering, Hiroshima University, \\ 1-4-1, Kagamiyama, Higashi-Hiroshima, Japan \\ Tel: +81-82-424-7703 Fax: +81-82-422-7024 \\ \{dhiro,mkatsumi,takahasi\}@hiroshima-u.ac.jp \\ WWW home page: http://www.pel.sys.hiroshima-u.ac.jp
}

\begin{abstract}
In a "Self-Balancing Production Line", each worker is assigned work dynamically, thus they can keep the balanced production under satisfying the specific conditions. For structure of line, in-tree assembly network line has been analyzed in previous paper. In that paper, line are virtually integrated to one and slowest to fastest sequence can be balanced under the integrated line. However, if an item consists of multiple parts and parallel work is possible, a new approach is applicable under the condition, and performance measure increase comparing to integrated line. In this paper, new integrated approach for both previous self-balancing line and buffer is proposed, and we compare the line that had been proposed in the previous paper.
\end{abstract}

\section{Keywords}

Self-balancing, production, blocking, multiple parts

\section{Introduction}

In the traditional assembly line, each worker is usually assigned to a fixed work, and each worker iterates the assigned work continuously as assembly line balancing. For this line, assigning workers to the balanced work is studied in the previous research, (for example, [1]). When imbalance of speed of workers exists in this kind of line, the slowest worker will delay the overall work. As a result, the production rate of the production line will also decrease. For solving this problem, "Self-Balancing Production Line" was introduced. The utilization of the mentioned method is reported in at least two commercial environments: apparel manufacturing and distribution warehousing [2]. In this type of production line, each worker is assigned 
to work dynamically, and when the last worker completes an item, he/she walks back and takes over the next item from his/her predecessor. Then, the predecessor walks back, takes over the next item from his/her predecessor, and so on until the first worker walks back and starts a new item. Since faster workers are assigned more work in processing an item, and slower workers are less, they can keep the balance. For this line, it has been found that the maximum production rate can be achieved if the workers are sequenced from slowest to fastest [3]. Also, the other conditions for three workers have been found numerically by simulation [1], and the performance of production line with $n$ workers have been analyzed mathematically [4].

Only one paper related to this line with multiple parts has been published [5]. In that paper, if all the line are virtually integrated to one line and workers are sequenced to slowest to fastest, then they can keep the balance and the maximum production rate can be achieved. However, if an item consists of multiple parts and parallel work is possible, a new approach is applicable under the condition, and performance measure increase comparing to integrated line. In this paper, new integrated approach for both previous self-balancing line and buffer is proposed, and we compare the line that had been proposed in the previous paper. This targeted line is a special case for self-balancing production line. However, using this approach, if there are many lines (ex., to make upper and lower parts of an item for each line) in that factory, it can be applied easily, and can be achieved higher production rate with lower flow time.

This paper is organized as follows: In section 2, assumptions, characteristics of this production line, behavior and formulation of this model are derived. In section 3 , new rules for integrated approach are shown. In section 4, we compare and analyze the rules for integrated approach. Finally, concluding remarks is described in section 5 .

\section{The Production Line}

In this section, assumptions and workers' behavior are explained, and characteristics of this line are also shown.

\subsection{Assumptions}

In this research, the production line with the following assumptions as shown in Fig. 1 is considered.

1. There are multiple lines, and there is a buffer in middle of the whole line to stock materials/parts. Capacity for each buffer is infinity.

2. Each worker processes only one identical item sequentially.

3. Workers are sequenced from one to $n$ on production line, and each worker never passes over the upstream and downstream workers.

4. Worker processes his/her work while moves along the line, and worker $i$ processes at constant velocity $v_{i}$ in the production line. In this paper, a continuous line is considered. This is different from the previous papers $[2,3$, $5]$. 
5. When the last worker finishes processing an item, worker $n$ walks back to worker $n-1$ and takes over the next item from worker $n-1$. Then, worker $n-1$ walks back to worker $n-2$ and takes over the next item from worker $n-2$. Similarly, all workers walk back to their preceding worker and take over the next item from the preceding worker, and worker 1 introduces a new item into the system. The time required to walk back and take over is ignored.

6. The total length of line is 1 . Under this condition, the position of worker $i$ when he/she starts to process is given by $x_{i}$. Then, the position at iteration $t$ is defined as $x_{i}^{(t)}$. Note that $x_{1}{ }^{(t)}=0$ for any iteration $t$. This is because the first worker starts to process an item.

7. There is one over-lapping zone. Starting and terminal position for upstream side is defined as $A_{0}$ and $A_{1}$, for downstream side, $B_{0}$ and $B_{1}$, respectively. Also, $A_{1}=B_{0}$ for convenience, and moving time to another line is ignored.

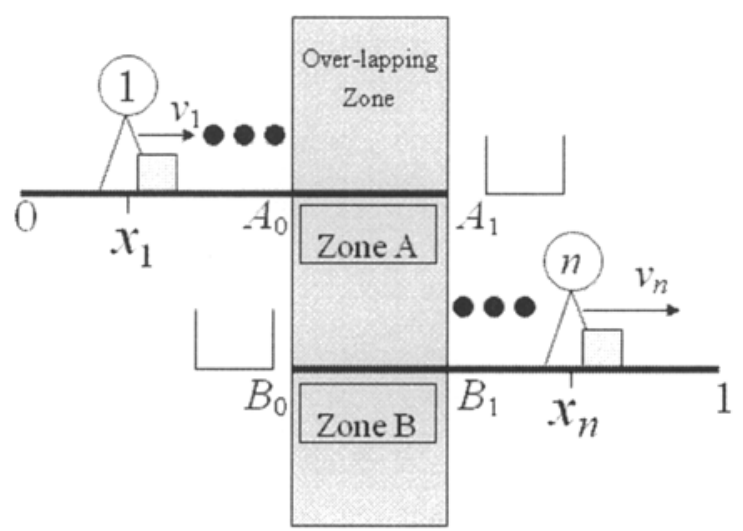

Fig. 1. Production line and position of $n$ workers.

\subsection{Self-Balancing and Convergence}

It has been proved that the production line can maintain balance when workers are sequenced from slowest to fastest [3]. Subsequently, the position of workers will converge to a unique fixed point defines as $x_{i}^{*}$ for worker $i$ as follows.

$$
x_{i}^{*}=\frac{\sum_{k=1}^{i-1} v_{k}}{\sum_{k=1}^{n} v_{k}}
$$

Under this condition, the production rate can be calculated as the sum of each worker's velocity $v_{i}(i=1,2, \ldots, n)$ of each worker. Hirotani et al. [4] find the convergence condition for $n$ workers as follows:

$$
\frac{\sum_{k=1}^{i-1}(-1)^{i+k-1}}{v_{n}}<1
$$

If this condition is satisfied for all workers, worker's starting point converges and the line can be balanced. 


\subsection{Imbalance}

When workers are not sequenced that the line can maintain balance, a slower worker prevents the preceding faster worker to continue processing. This condition is called blocking. After blocking occurs, the faster worker moves at the same velocity as the slower worker until the last worker finishes his/her item. In this condition, the position of workers will not converge to a fixed point, and thus, the production rate decreases. Two kinds of blockings exist, one is blocking caused by worker's initial position, and another is blocking caused by worker's velocity.

\subsection{Behavior and Formulation of the Model}

Fig. 2 shows the time chart for three workers. In Fig. 2, horizontal axis represents worker's position and vertical axis represents time. Zero at the horizontal axis means the head of line and one shows the end of line. Since each worker works with changing his/her position down-stream, diagonal lines, according to their speeds, can represent the worker that is working. Also, since the time to walk back and take over is ignored, a horizontal line can represent the worker that is walking back and taking over. When worker 3 finishes an item, i.e. the position is one, he/she walks back to worker 2 and takes over the next item from worker 2 . At the same instant, worker 2 walks back to worker 1 and takes over the next item from worker 1 . Then, worker 1 walks back and the position reaches zero, where he/she starts to process a new item.

The position of worker $i$ at iteration $t$ is defined as $x_{i}^{(r)}$, and the worker $i$ 's velocity is defined as $v_{i}$. Using these notations, when no blocking occurs, position of the worker at one iteration changes, as follows:

$$
\left\{\begin{array}{l}
x_{1}^{(t+1)}=0 \\
x_{i}^{(t+1)}=x_{i-1}{ }^{(t)}+v_{i-1}\left(\frac{1-x_{n}{ }^{(t)}}{v_{n}}\right) \quad(i=2,3, \cdots, n)
\end{array}\right.
$$

Iteration time is derived by calculating the time spent in one iteration. Iteration time of each worker $a_{i}^{\left({ }^{\prime}\right)}$ is shown, as follows:

$$
a_{i}^{(t)}=\frac{x_{i+1}{ }^{(t)}-x_{i}^{(t)}}{v_{i}} \quad(i=1,2, \cdots, n)
$$

Using this, the production rate can be calculated as the reciprocal of the iteration time, as follows:

$$
\min _{i}\left\{1 / a_{i}^{(t)}\right\} \quad(i=1,2, \ldots, n)
$$

It should be note that when no blocking occurs, the iteration time of each worker $i$ is the same.

In Fig. 2, at the square area, blocking only caused by workers 1 and 2 . After the blocking, worker 1 processes at the same velocity as slower worker 1, until worker 3 finishes an item. Therefore, iteration time increases a lot because of slower worker 2 , and the production rate decreases. If blocking does not occur, self-balancing and 
convergence of positions of each worker is obtained. Thus, the production achieves the maximum production rate.

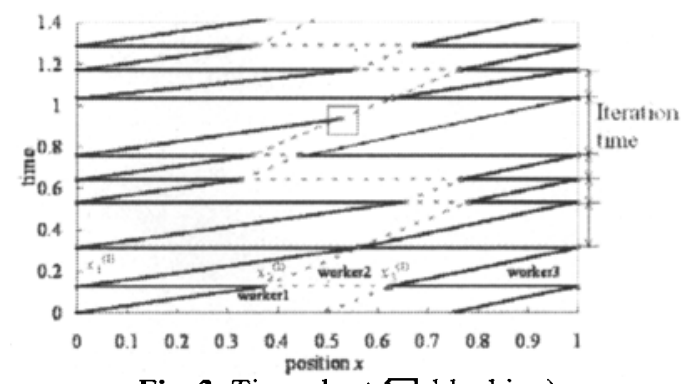

Fig. 2. Time chart $\square$ :blocking).

\section{New Worker's Behavior Rule}

Considering integrated approach, rule for worker's behavior should be defined. Therefore, we propose two new worker's behavior rules in this paper. For these rules, performance measures are better than that of derived by previous papers [2-5]. Worker's behavior changes in the over-lapping zone as follows.

- Behavior of worker when processing

$>\quad$ When any worker $i$ finishes processing until $A_{1}$, he/she goes to $B_{0}$, and starts to process to remaining work unless worker $i+1$ walks back.

$>$ When worker finishes processing in zone A, he/she puts an item in buffer and walks back to preceding worker.

$>$ When worker who finishes processing in zone B, he/she takes an item in buffer and continues to process. If there is no item in buffer, he/she must wait until an item is put in buffer.

- Behavior of worker when walking back

$>\quad$ When worker $i$ takes over an item to worker $i+1$, worker $i$ walks back to worker $i-1$ and takes over an item from worker $i-1$ and starts to process the item if worker $i-1$ is in the downstream from $B_{0}$.

$>\quad$ When worker $i$ takes over an item to worker $i+1$, worker $i$ walks back to $B_{0}$ and starts to process a new item if worker $i-1$ is between $A_{0}$ and $A_{1}$.

$>\quad$ When worker $i$ takes over an item to worker $i+1$, worker $i$ follows one of two rules if worker $i$ is in the upstream from $A_{0}$. These rules are shown in following subsections.

\subsection{Rule 1 (Zone Rule: ZR)}

This rule is that when worker $i$ takes over an item to worker $i+1$, worker $i$ walks back to worker $i-1$, and takes over an item from worker $i-1$ if worker $i$ is in the upstream from $A_{0}$. In applying this rule, advantage is that not only time for completing an item is smaller than that of the previous researches [2-5] but also the maximum 
production rate maintains the same as previous researches by processing at the same time in the over-lapping zone.

\subsection{Rule 2 (Fixed Rule: FR)}

This rule is that when worker $i$ takes over an item to worker $i+1$, worker $i$ walks back to $B_{0}$ if worker $i$ is in the upstream from $A_{0}$. In applying this rule, advantage is that not only it is easy to balance by separating the working area since there is a fewer worker in one line but also time for completing an item is smaller than that of the previous researches $[2-5]$.

\section{Comparison and Analysis}

In this section, we compare two rules for integrated approach. In previous researches [2-5], only production rate is considered as performance measure. However, in this paper, flow time should be considered since an item consists of multiple parts, and thus an item can be made earlier than integrated line. Therefore, we use two measurements: production rate and flow time. Flow time means that time for completing an item. If flow time is low, an item is completed earlier. We use two figures to explain. Fig. 3(a) and (b) are examples of time chart for three workers $\left(v_{1}=1, v_{2}=3, v_{3}=5\right.$ ). For these Figures, $A_{0}=0.4, A_{1}=B_{0}=0.5, B_{1}=0.6$. Fig. $3(\mathrm{a})$ is for ZR and (b) is for FR.

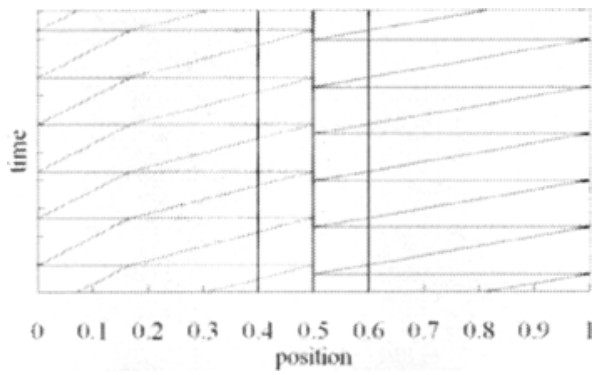

(a) ZR (Zone Rule)

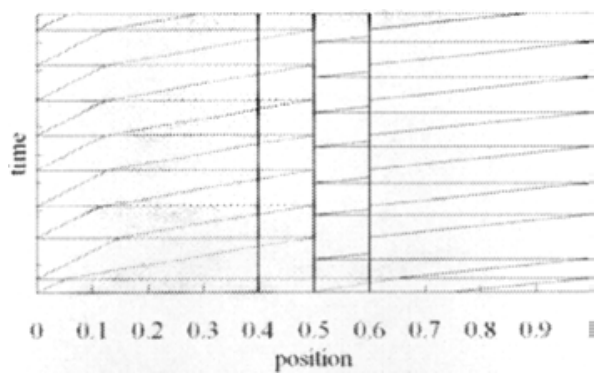

(b) FR (Fixed Rule)

Fig.3. Example of time chart of steady-state for three workers with multiple parts 


\subsection{Production Rate (PR)}

First, we analyze production rate. In Fig. 3(a), two workers process in the overlapping zone alternatively. In previous rule, if the line is balanced, all the workers process the same area in steady-state. However, in Fig. 3(a), slower worker do not processes the same area at any iteration. In stead, down-stream faster worker processes. Under this phenomenon, the production rate is the same as maximum because a faster worker can make up for a slower worker. On the other hand, there is a possibility that production rate for ZR is less than that of FR. In Fig. 3(b), the fastest worker cannot process more work because of rule for FR. This is why production rate is not maximum (sum of the velocity of all workers) unless the line can be balanced. This is shown defined as $P R_{Z R}$ using equation (4) and (5) as follows:

$$
P R_{Z R}=\min _{i}\left\{\frac{x_{i+1}^{*}-x_{i}^{*}}{v_{i}}\right\}
$$

where,

$$
x_{i}^{*}=\left\{\begin{array}{cc}
A_{1} \cdot \frac{\sum_{k=1}^{i} v_{k}}{\sum_{k=1}^{l} v_{k}} & \text { (if } i \leq l) \\
\left(1-A_{1}\right) \cdot \frac{\sum_{k=1}^{l} v_{k}}{\sum_{k=l+1}^{n} v_{k}} & \text { (if } i>l)
\end{array}\right.
$$

Equation (7) is different with equation (1). Equation (7) indicates each worker works according to their working velocity in each line while each worker works according to their working velocity in one previous line.

Note that if faster worker in the downstream from $B_{0}$ exists, there is a high possibility that item is empty in buffer. Therefore, the worker must wait until an item is put in buffer, and thus production rate decreases because of waiting time. On the other hand, if slower worker in the downstream from $B_{0}$ exists, this problem does not occur.

\subsection{Flow Time (FT)}

Next, we compare and analyze flow time. In fig.3(a), at any iterations, worker 2 finishes to process in zone A, at the same time, worker 3 finishes to process in zone B. This means that in flow time, processing in zone B can be neglected, and thus flow time is smaller than previous research. On the other hand, in fig.3(b), worker 3 must wait because of no items in buffer. However, flow time is shorter than that of derived in previous research because worker 3 can start to process in zone B while 
worker 2 processes in zone A. Comparing these results, in flow time, FR is better than ZR. Flow time for ZR and FR defined as $F T_{Z R}$ and $F T_{F R}$ can be calculated as follows:

$$
\begin{gathered}
F T_{Z R}=n /\left(\frac{n}{\sum_{i=1}^{n} v_{1}}-\frac{B_{1}-B_{0}}{v_{i}}\right) \\
F T_{F R}=n /\left(\sum_{i=1}^{n} \frac{v_{i}}{x_{i}^{*}}-\frac{B_{1}-B_{0}}{v_{i}}\right)
\end{gathered}
$$

where, $x_{i}^{*}$ is shown in equation (7). Above equations mean that time for overlapping zone can be neglected. Therefore, flow time is smaller than that of previous research.

\section{Concluding Remarks}

In this paper, we propose integrated approach that combine buffer and traditional self-balancing line approach for self-balancing production line with multiple parts. Considering integrated approach, two rules (Zone rule and Fixed rule) are proposed. As a result, maximum production rate is the same as previous research, and if worker have to wait, production rate decreases according to waiting time. On the other hand, flow time is smaller than that of previous research. This result indicates that an item can complete earlier.

In our research, we assume there is one over-lapping zone. Considering multiple over-lapping zones is future research works.

\section{References}

[1] A. Scholl, Balancing and Sequencing of Assembly Lines, (Physica-Veriag, New York, 1995).

[2] J. J. Bartholdi, and L. A. Bunimovich, Dynamics of two- and three-worker "Bucket Brigade" production lines. Oper. Res., 47(3), 488-491 (1999).

[3] J. J. Bartholdi, and D. D. Eisenstein, A production line that balances itself. Oper, Res., 44(1), 21-34 (1996).

[4] D. Hirotani, Myreshka, K. Morikawa, and K. Takahashi, Analysis and design of the self-balancing production line, Computers and Industrial Engineering, 50(4), 488-502 (2006).

[5] J. J. Bartholdi, and D. D. Eisenstein, Bucket Brigades on In-tree Assembly Networks, European Journal of Operational Research, 168, 870-879 (2006). 